\title{
ERRATUM
}

\section{Erratum to: Provider patient-sharing networks and multiple-provider prescribing of benzodiazepines}

\author{
Mei-Sing Ong, $P h D^{1,2}$, Karen L. Olson, $P h D^{1,3}$, Aurel Cami, $P h D^{1,3}$, Chunfu Liu, PhD ${ }^{5}$, Fang Tian, PhD ${ }^{5}$, \\ Nandini Selvam, $P h D M P H^{5}$, and Kenneth D. Mandl, MD, MPH ${ }^{1,3,4}$

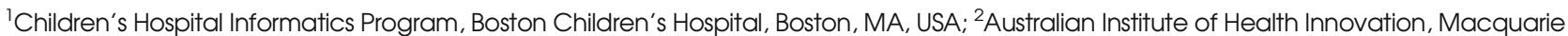 \\ University, Sydney, NSW, Australia; ${ }^{3}$ Department of Pediatrics, Harvard Medical School, Boston, MA, USA; ${ }^{4}$ HealthCore, Inc., Alexandria, VA, USA; \\ ${ }^{5}$ Center for Biomedical Informatics, Harvard Medical School, Boston, MA, USA.
}

The odds ratio for the relationship between median care density and multiple prescribing of benzodiazepine was incorrectly reported in the text as $0.76(95 \%$ CI $0.60-0.95$; $\mathrm{p}=0.028$ ).

It should have read, "Backward stepwise logistic regression revealed that median care density was negatively associated with the risk of an overlapping benzodiazepine prescription (OR 0.76; 95\% CI $0.60-0.95 ; \mathrm{p}=0.0178$ ) after adjusting for covariates (Table 3)." In the Discussion, it should have also read the following: "Consistent with these studies, in univariate analysis, we showed a significant relationship between the number of prescribers and overlapping benzodiazepine prescriptions; however, the magnitude of the association was reduced when considered alongside care density in multivariate analysis."

The online version of the original article can be found at http://dx.doi.org/ 10.1007/s11606-015-3470-8.

Published online October 30, 2015 